DOE/ER/40561

\title{
Measuring Strangeness Matrix Elements of the Nucleon
}

\author{
E.M. Henley \\ Institute for Nuclear Theory, HN-12 and Department of Physics, FM-15 \\ University of Washington, Seattle, Washington 98195, USA \\ G. Krein \\ Department of Physics, FM-15, University of Washington \\ Seattle, Washington 98195, USA \\ and Instituto de Fisica Teorica, Sao Paulo, Brazil \\ S.J. Pollock \\ Institute for Nuclear Theory, HN-12, University of Washington \\ Seattle, Washington 98195, USA \\ A.G. Williams \\ Department of Physics, FM-15, University of Washington \\ Seattle, Washington 98195, USA \\ and Physics Department and Supercomputer Research Institute \\ Florida State University, Tallahassee, Florida 32306-3016, USA
}

\section{PREPARED FOR THE U.S. DEPARTMENT OF ENERGY}

This report was prepared as an account of work sponsored by the United States Government. Neither the United States nor the United States Department of Energy, nor any of their employees, nor any of their contractors, subcontractors, or their employees, makes any warranty, express or implied, or assumes any legal liability or responsibility for the product or process disclosed, or represents that its use would not infringe privately-owned rights.

By acceptance of this article, the publisher and/or recipient acknowledges the U.S. Government's right to retain a nonexclusive, royalty-free license in and to any copyright covering this paper.

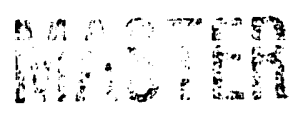




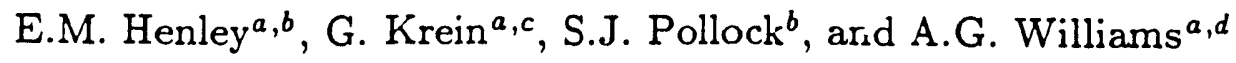 \\ ${ }^{a}$ Department of Physics, FM-15, ${ }^{b}$ Institute for Nuclear Theory, HN-12 \\ University of Washington Seattle, Washington 98195 \\ 'Instituto de Física Téorica, São Paulo, Brazil \\ ${ }^{d}$ Physics Department and Supercomputer Research Institute* \\ Florida State University, Tallahassee, FL 32306-3016
}

\begin{abstract}
Experiments are proposed to measure various strangeness matrix elements of the nucleon. Examples are electro- and neutrino-production of phi mesons and the difference between neutrino and antineutrino scattering from isospin zero targets, e.g., deuterons.
\end{abstract}

* Present and permanent address 
There has been a great deal of recent interest in the strange quark matrix elements of the proton. Deductions of the sigma-term ${ }^{1)}$ from pion-nucleon scattering and of the proton spin structure function ${ }^{2)}$ from the EMC measurements both suggest a sizeable strange quark presence in the nucleon. Since this conclusion is contrary to the generally accepted belief that the low-energy structure of the nucleon is well described by three valence $(u$ and $d$ ) quarks, there have been numerous theoretical interpretations of the experimental findings that do not require any, or only a tiny fraction of strangeness in the proton. ${ }^{3)}$ By contrast, there are only a few publications that suggest measurements of the strangeness content, or ratiner strangeness matrix elements, in the nucleon ${ }^{4-7)}$ In this Letter we outline some measurements that allov. the determination of strangeness matrix elements in the nucleon.

The OZI rule suggests that the coupling of the strange quarks to rucleons should be very small in the absence of strangeness in the nucleons. Ellis, Gabathuler, and Karliner ${ }^{4}$ noted that the OZI rule appears to be violated in phi production from $\bar{p} p$ and $p p$ collisions. Since the $\phi$ is a pure $(s \bar{s})$ vector meson, experiments such as $\bar{p} p \rightarrow \phi \pi^{+} \pi^{-}$ measure the vector matrix elements $\leftarrow N\left|\bar{s} \gamma^{\mu} s\right| N>$. Beck, McKeown, and others ${ }^{5)}$ have proposed parity-violating elastic electron scattering from hydrogen to measure this matrix element. We suggest that electroproduction of $\phi$ mesons from a hydrogen target, e $p \rightarrow e p \phi$, can also be used. ${ }^{7)}$ It has the advantage that the interaction of the electron with the proton has been studied extensively. On the other hand, there is a background due to the diffractive production of $\phi$ 's via vector meson dominance. ${ }^{8)}$

In this mechanism the photon is transformed into a phi meson which scatters diffractively (Pomeron exchange) off the target. It should be possible to distinguish these two production cross sections on the basis of their energy dependence, their angular distributions, and in polarization measurements. Previous measurements ${ }^{9)}$ are not sufficiently accurate to determine the mechanism and do not rule out the knockout process due to 
the presence oi sirangeness in the nucleon.

We are currently carrying out a detailed calculation of the knockout process in a constituent quark model, and are comparing it to the prediction of the VMD model near the phi-production threshold. We have examined the energy-dependence of our knockout model for a $20 \%$ admixture of strange quarks in the nucleon. As a funct $n$ of the beam energy the total cross-section rises more rapidly and to a greater value than the relatively smooth and slowly rising VMD background. We have also found that in the laboratory frame the knockout cross-section has a clear minimum when the three-momentum transfer is parallel or antiparallel to the beam direction and a maximum when the two are orthogonal. This feature appears insensitive to the details of the model and follows primarily from the vector nature of the electromagnetic current and from the knockout nature of the process. The diffractive VMD process does not have this feature. We smilarly expect that a comparison of predictions for polarized cross-sections will assist in unfolding these two mechanisms. This work will be reported elsewhere.

The pseudoscalar matrix element $\left\langle p\left|\bar{s} \gamma^{5} s\right| p>\right.$ is more difficult to obtain. The $\eta$ and $\eta^{\prime}$ both contain strange mesors, and it would be necessary to know the mixing angle well and carry out a difference measurement to obtain this matrix. The GoldbergerTreiman relation does not hold because of the axial anomaly. ${ }^{10}$ )

The measurement of the interesting "spin" matrix element $\left\langle N\left|\bar{s} \gamma^{\mu} \gamma^{5} s\right| N>\right.$ is possible through a variety of experiments. If there is no strangeness axial-vector matrix element, then the Weinberg-Salam theory (at the tree level) only has an SU(2) isovector matrix element, $\bar{u}_{N} \frac{1}{2} \tau^{(3)} \gamma^{\mu} \gamma^{5} u_{N} F_{A}^{(3)}\left(Q^{2}\right)$, with $F_{A}^{(3)}(0)=1.26$. Due to the mismatch of the the strong and weak isospin for the s-quark, a small induced $\mathrm{SU}(2)$ isoscalar matrix element 


$$
\bar{u}_{N} \gamma^{\mu} \gamma^{5} u_{N} F_{A}^{(0)} \propto<p\left|\bar{s} \gamma^{\mu} \gamma^{5} s\right| p>
$$

occurs, with $F_{A}^{(0)}(0) \approx 0.05 .{ }^{11)}$ Since very little is known abcut this matrix element, or its $\mathrm{SU}(3)$ counterpart, $\tilde{F}_{A}^{(0)},{ }^{5-7)}$ (we use a superscript $\sim$ for $\mathrm{SU}(3)$ form factors) it is clearly of great interest to determine its magnitude from other experiments than the EMC measurements. ${ }^{2)}$ There are a number of ways to obtain $F_{A}^{(0)}$. In the past, parity-violating elastic scattering of electrons on deuterons has been proposed by us and colleagues. ${ }^{7,12,13)}$ If the scattering is carried out with longitudinally polarized electrons, then backward scattering is required. ${ }^{13)}$ A more direct, but also more difficult experiment is to polarize the deuteron (or ${ }^{6} \mathrm{Li}$, or other isospin zero, spin 1) target or to measure the polarization of the final deuteron in the scattering plane. ${ }^{11)}$ At small $q^{2}$ the resulting parity-violating asymmetry vanishes if $F_{A}^{(0)}=0$ and is directly proportional to it, or to $\tilde{F}_{A}^{(s)}=\tilde{F}_{A}^{(0)}-\frac{2 \sqrt{3}}{3} \tilde{F}_{A}^{(8)}$ in terms of form factors proportional to $\mathrm{SU}(3)$ generators. The asymmetry is small, ${ }^{12,13)}$ about $10^{-5} F_{A}^{(0)}\left|q^{2}\right| / M^{2}$, where $q^{2} \equiv-Q^{2}$ is the squared momentum transfer and $M$ is the nucleon mas's, because the weak vector current of the electron is proportional to $\left(1-4 \sin ^{2} \theta_{W}\right) \approx 0.08$, with $\theta_{W}$ the Weinberg angle.

To circumvent this smallness, we propose here the use of neutrino scattering. Ahrens et al. ${ }^{14)}$ have measured the elastic scattering of neutrinos and antineutrinos from hydrogen. Their result suggests $F_{A}^{(0)} \approx 0.08 \pm 0.04 .{ }^{6), 14)}$ A more direct experiment is the excitation of a $1^{+}$state of isospin 0 in an even-even light nucleus such as $\left.{ }^{12} \mathrm{C},{ }^{15}\right)$ or the measurement of elastic neutrino and antineutrino scattering from an isospin zero target, e. g., ${ }^{2} \mathrm{H},{ }^{4} \mathrm{He},{ }^{6} \mathrm{Li}$. (In the scattering from ${ }^{2} \mathrm{H}$, the target recoil could be measured to ascertain that the scattering is elastic.) The difference in cross sections

$$
\sigma(\nu d \rightarrow \nu d)-\sigma(\bar{\nu} d \rightarrow \bar{\nu} d)
$$


is a direct measure of $F_{A}^{(0)}$. The difference in the neutrino and antineutrino cross sections is proportional to the interference between the vector and axial vector matrices. The beam spectra could be normalized by measuring total cross sections on a target such as ${ }^{12} \mathrm{C}$. The vector matrix is known from CVC, aside from a possible modification induced by strangeness and measured by the deviation of $\tilde{F}_{2}^{(0)}$ from $\left(\frac{2 \sqrt{3}}{3} \tilde{F}_{2}^{(8)}-\left(\kappa_{p}+\kappa_{n}\right)\right)$; the difference in cross sections vanishes if $F_{A}^{(0)}=0$.

In impulse approximation, the difference in cross sections is given by

$$
\begin{aligned}
& N \equiv \frac{1}{2}\left[\frac{d \sigma}{d Q^{2}}(\nu d \rightarrow \nu d)-\frac{d \sigma}{d Q^{2}}(\bar{\nu} d \rightarrow \bar{\nu} d)\right] \\
& N=\frac{4}{3} \frac{G_{F}^{2} M}{\pi E} \eta(1-\zeta) F_{A}^{(0)}\left(F_{1}^{(0)}+F_{2}^{(0)}\right) F_{D},
\end{aligned}
$$

where $\eta=\frac{Q^{2}}{4 \Lambda \Lambda^{2}}, \zeta=\frac{Q^{2}}{8 \Lambda L E}, F_{1}^{(0)}(0)=-2 \sin ^{2} \theta_{W} \approx-0.46, F_{2}^{(0)}(0)=-2 \sin ^{2} \theta_{W}\left(\kappa_{p}+\right.$ $\left.\kappa_{n}\right)-\tilde{F}_{2}^{(s)}, \approx-0.055-\tilde{F}_{2}^{(s)}, F_{A}^{(0)}$ is the unknown axial form factor, $F_{D}$ is the (here we assume a single form factor for simplicity) deuteron form factor, $E$ is the neutrino (antineutrino) energy, $M$ is the mass of the nucleon, and $G_{F}$ is the weak Fermi coupling constant. We assume dipole form factors, $F_{1} \propto F_{2} \propto\left(Q^{2}+m_{V}^{2}\right)^{-2}$ and $F_{A} \propto\left(Q^{2}+\right.$ $\left.m_{A}^{2}\right)^{-2}$, with $m_{V} \approx 0.84 \mathrm{GeV}, m_{A} \approx 1.03 \mathrm{GeV},{ }^{14)}$ even though the isoscalar axial form factor could differ from the isovector one. For small $Q^{2}$ the difference in cross sections, $N$, increases linearly with the absolute value of the squared momentum transfer, $Q^{2}=$ $-q^{2}$, but as $Q^{2}$ increases, the nucleon and nuclear (e.g. deuterium) form factors all fall and thus the counting rate becomes small. For instance, for $Q^{2} \approx M^{2}$, we have $Q^{2} R_{D}^{2} \approx 400$, where $R_{D}$ is the deuteron radius $\approx 4.3 \mathrm{f}$, and the deuterium form factor is $\lesssim 10^{-2}$.

We define the ratio $R$

$$
R \equiv \frac{\frac{d \sigma}{d Q^{2}}(\nu d \rightarrow \nu d)-\frac{d \sigma}{d Q^{2}}(\bar{\nu} d \rightarrow \bar{\nu} d)}{\frac{d \sigma}{d Q^{2}}(\nu d \rightarrow \nu d)+\frac{d \sigma}{d Q^{2}}(\bar{\nu} d \rightarrow \bar{\nu} d)} .
$$

For $Q^{2} \gtrsim 0.5(\mathrm{GeV} / \mathrm{c})^{2}$ it is not sufficiently accurate to consider a single form factor for the deuteron. $R$ is approximately proportional to $\eta$ for $Q^{2} \lesssim 0.5 \mathrm{GeV}^{2}$; its magnitude is 
sizeable $(\gtrsim 0.1)$ for $Q^{2} \gtrsim 0.3(\mathrm{GeV} / \mathrm{c})^{2}$. However, at large values of $Q^{2}$ the differences of the various deuteron form factors matter and for $Q^{2} \gtrsim M^{2}$ the differential cross sections and $N$ become exceedingly small, so that the measurement is very difficult. $N$ reaches a maximum at $Q^{2}$ just under $0.1(\mathrm{GeV} / \mathrm{c})^{2}$. In Fig. 1, we show $|R|$ as a function of $Q^{2}$ for $E=M c^{2}$ and $F_{A}^{(0)}(0)=0.2$, for $\tilde{F}_{2}^{(s)}(0)=0$ and \pm 0.2 . The graphs of Fig. 1 and Eq. (2) show that both $F_{A}^{(0)}(0)$ and $\tilde{F}_{2}^{(s)}(0)$ might be obtained if $R$ is measured as a function of $Q^{2}$ and $E$. We believe that this experiment is feasible at a variety of energies (e.g., at LAMPF). Another possible experiment is the elastic scattering of either $\nu$ 's or $\bar{\nu}$ 's from an isospin zero polarized target, or the measurement of the longitudinal polarization of the recoil.

A further experiment is $\phi$ production by $\nu p \rightarrow \nu p \phi$. This experiment is sensitive to both $\left\langle p\left|\bar{s} \gamma^{\mu} s\right| p>\right.$ and $\left\langle p\left|\bar{s} \gamma^{\mu} \gamma^{5} s\right| p>\right.$. Just as for the electroproduction of phi's, the neutrino production will have a VMD diffractive background. In parallel with the previously mentioned electroproduction work we are pursuing the same approach to resolving these two competing production mechanisms. One difference is that the angular distribution for the knockout mechanism now has a more complicated form due to the additional axial-vector coupling of the $Z^{0}$ to the struck $s$ or $\bar{s}$ quark.

In summary, we have proposed a number of nuclear experiments for measurements of the unknown $\tilde{F}_{2}^{(0)}$ and $F_{A}^{(0)}$. Details will appear elsewhere.

One of the authors (EMH) thanks Wick Haxton for a helpful discussion. 
1. T.P. Cheng and R.F. Dashen, Phys. Rev. Lett. $\underline{26}$ (1971) 594 and Phys. Rev. D $\underline{13}$ (1976) 216; T.P. Cheng, Phys. Rev. D $\underline{13}$ (1973) 2161; C.A. Dominguez and P. Langacker, Phys. Rev. D $2 \underline{4}$ (1981) 190; J. Donoghue, Ann. Rev. Nucl. Part. Sci. $\underline{39}(1989) 1$.

2. J. Ashman et al., Phys. Lett. B206 (1988) 364 and Nucl. Phys. B $\underline{328}$ (1989) 1.

3. M.A. Nowak, J.J.M. Verbaarshot and I. Zahed, Phys. Lett. B217 (1989) 157; H.

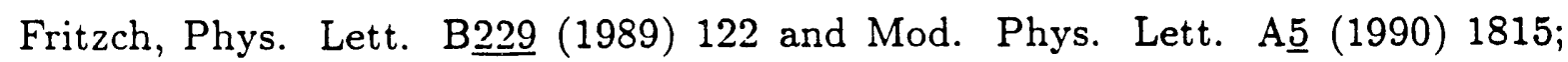
R. Anselmino and M.D. Scadron, Phys. Lett. B229 (1989) 117; J. Stern and G. Cle'ment, Phys. Lett. B231 (1989) 471; V. Bernard and U. Meissner, Phys. Lett. $\underline{216}$ (1989) 392 and $\underline{223}$ (1989) 439; R.L. Jaffe and A. Manohar, Nucl. Phys. B $\underline{337}$ (1990) 509.

4. J. Ellis, Gabathuler, and M. Karliner, Phys. Lett. B217 (1989) 173.

5. D. Beck, Phys. Rev. D 39 (1989) 3248; R. Decker and Th. Leize, Phys. Lett. B216 (1990) 233; Fayya Zuddin and Ria Zuddin, Phys. Rev. D吕 (1990) 794; R.D. Mckieown, Phys. Leit. B219 (1989) 140; E.J. Beise and R.D. McKeown, Comm. Nucl. Part. Phys. 20 (1991) 105; J. Napolitano, Phys. Rev. C $\underline{43}$ (1991) 1473.

6. D. Kaplan and A.V. Manohar, Nucl. Phys. B 310 (1988) 527; B.R. Holstein, Weak Interactions in Nuclei, Princeton Univ. Press, Princeton, N.J., 1989, pp 96-97.

7. E.M. Henley, G.Krein, and A.G. Williams, Bull. Am Phys. Soc. $\underline{34}$ (1989) 1828.

8. See e.g., M.S. Chen, F.S. Henyey, and G.L. Kane, Nucl. Phys. B118 (1977) 345.

9. R. Dixon et al., Phys. Rev. D $\underline{19}$ (1979) 3185; D.G. Cassel et al., Phys. Rev. D $\underline{24}$ (1981) 2787 .

10. R.L. Jaffe, Phys. Lett. B 193 (1987) 101; T. Kunihiro and T. Hatsuda, Phys. Lett.

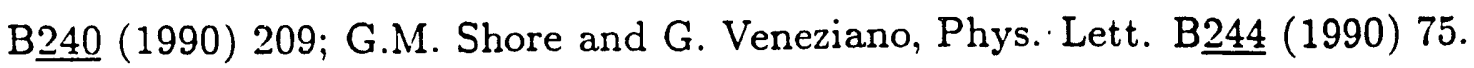


11. J. Collins, F. Wilczek, and A. Zee, Phys. Rev. D $\underline{18}$ (1978) 242; L. Wolfenstein, Phys. Rev. D 19 (1979) 3450.

12. W-Y.P. Hwang and E.M. Henley, Ann. Phys. $\underline{129}$ (1980) 47.

13. T. Frederico, E.M. Henley and G.A. Miller, submitted for publication; S.J. Pollock, Phys. Rev. D $\underline{42}$ (1990) 3010; erratum,Phys. Rev. D $\underline{43}$ (1991) 2447.

14. L. Ahrens et al., Phys. Rev. D $\underline{35}$ (1987) 785.

15. T. Suzuki, Y. Kohyama, and K. Yazaki, Phys. Lett. B252 (1990) 323. 


\section{Figure Caption}

Figure 1. Plot of $R$ as a function of $Q^{2}$ for $Q^{2} \lesssim 0.5(\mathrm{GeV} / \mathrm{c})^{2}$, for an s-wave deuteron. The solid curve is for $F_{2}^{(s)}=0$, the dashed one for $F_{2}^{(s)}=-0.2$, and the dotted one for $F_{2}^{(s)}=+0.2$. 


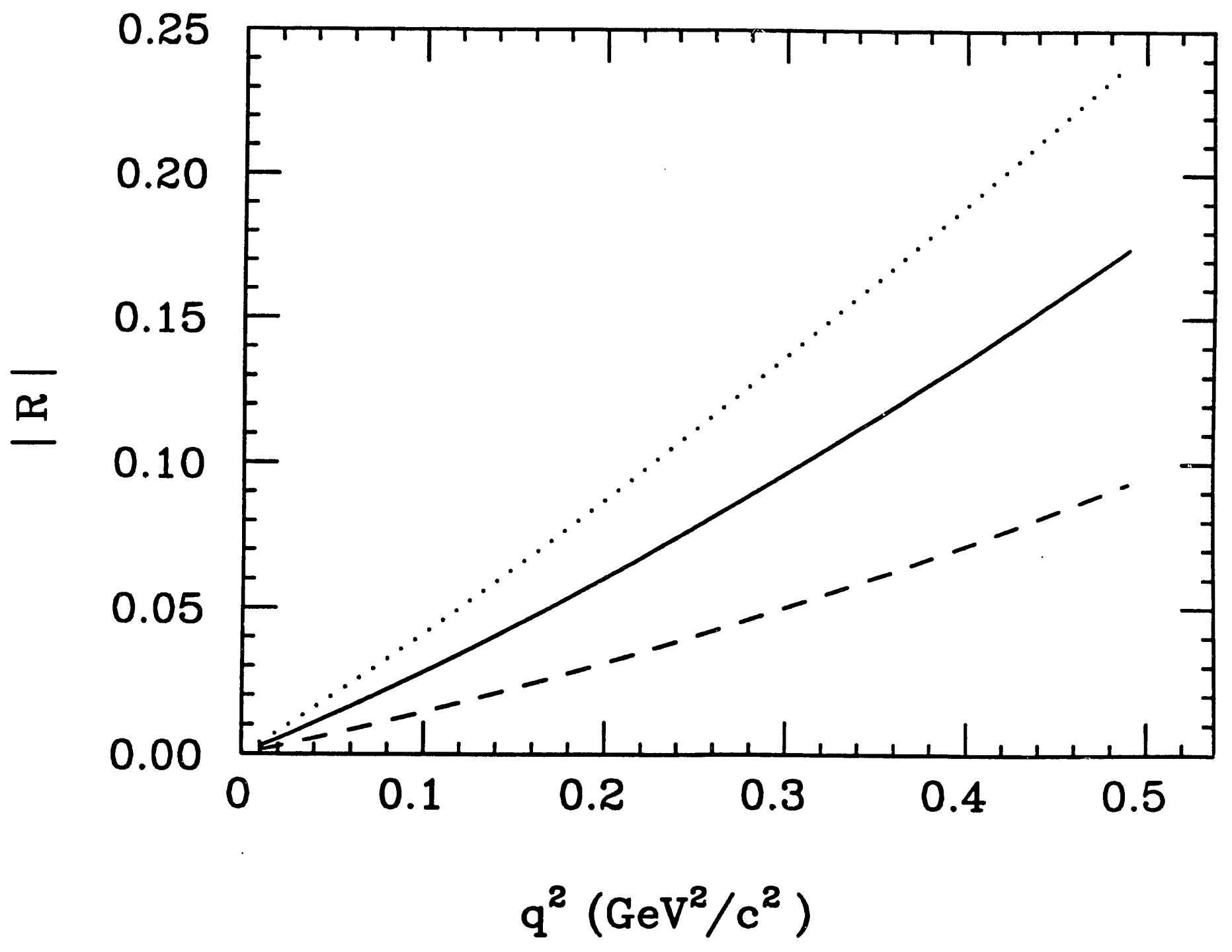

Figure 1 

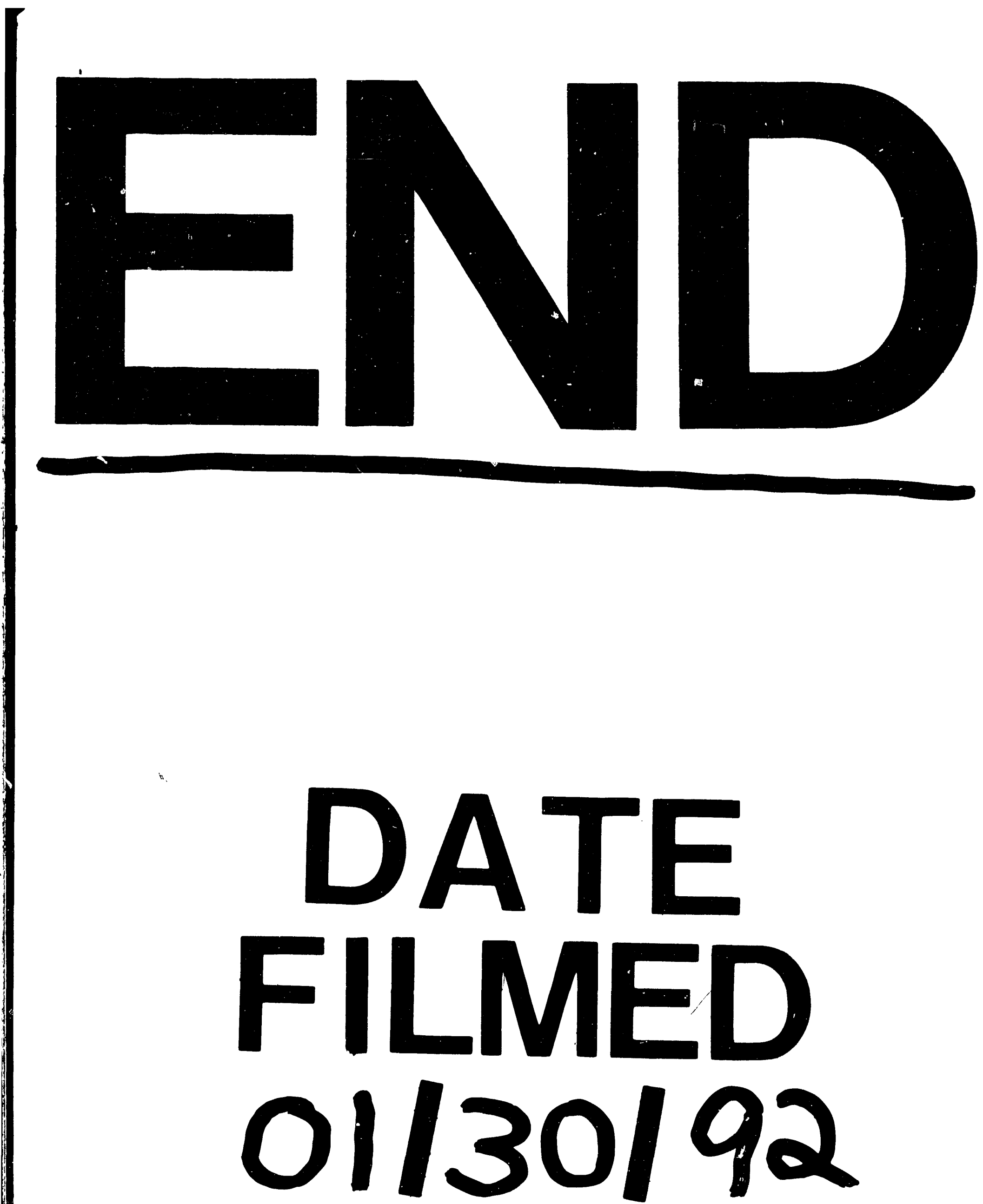